\title{
100G PAM-4 PON with 34 dB Power Budget Using Joint Nonlinear Tomlinson-Harashima Precoding and Volterra Equalization
}

\author{
Lei Xue ${ }^{(1)(2)}$, Rui Lin(1), Joris Van Kerrebrouck ${ }^{(3)}$, Lilin Yi(2), Jiajia Chen ${ }^{(1)}$, Xin Yin ${ }^{(3)}$ \\ (1) Chalmers University of Technology, S-412 96 Göteborg, Sweden, leixu@chalmers.se \\ (2) State Key Laboratory of Advanced Optical Communication System and Networks, Shanghai Jiao \\ Tong University, Shanghai, China, lilinyi@sjtu.edu.cn \\ (3) Ghent University - IMEC, IDLab, Gent 9000, Belgium, Department of Information Technology, \\ xin.yin@ugent.be
}

\begin{abstract}
We experimentally demonstrate 100G PAM-4 passive optical network using DML-based intensity modulation and direct detection with 3-dB system bandwidth of $15 \mathrm{GHz}$ in O-band. Combining nonlinear Tomlinson-Harashima precoding at the transmitter and 2nd-order Volterra at the receiver enables 34-dB power budget for PON downstream.
\end{abstract}

\section{Introduction}

To cope with the ever-increasing bandwidth demand, the international standardization organizations IEEE 802.3ac and ITU-T Q2/SG15 groups are going to release the solutions for 25 $\mathrm{Gbps} / \lambda$ and $50 \mathrm{Gbps} / \lambda$ passive optical networks $(P O N s)^{[1]-[2]}$, while the single channel 100G PON is currently being investigated in research community ${ }^{[3-8]}$. Directly modulated later (DML) with intensity modulation and direct detection (IMDD) is often considered more cost-effective for PON compared to external modulation and coherent detection ${ }^{[3]}$. Besides, due to the dispersion problem in C-band, O-band is preferred, particularly for high-speed systems. Nevertheless, higher fiber attenuation in O-band requires optical amplifiers. Regardless the dispersion, bandwidth limitation and nonlinearities from optoelectronics and fiber are the main problems need to be addressed for 100G PON for high power budget $(>29 \mathrm{~dB})$. To relax the requirement on high-bandwidth optoelectronic devices, 4-level pulse amplitude modulation (PAM-4) doubles spectrum efficiency, and hence is considered as a primary variant for 100G PON. Meanwhile, digital signal processing (DSP) algorithms, e.g., feedforward equalizer (FFE), decision feedback equalizer (DFE), Volterra filter, neural network (NN) are introduced to mitigate the linear and nonlinear distortions for 100G PON. However, these algorithms are often employed at the ONU side, which increases the deployment cost on a per-user basis.

In this paper, we propose to use joint equalization, i.e., nonlinear TomlinsonHarashima Precoding (NTHP) at the OLT side combined with 2nd-order Volterra at the ONU side to compensate linear and nonlinear distortions. We experimentally demonstrate 100G PAM-4 transmission over $20 \mathrm{~km}$ single

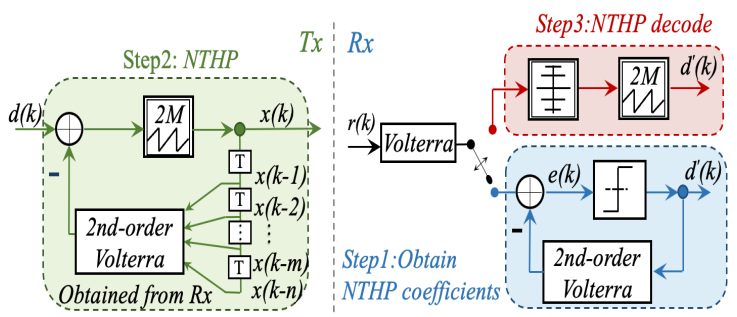

Fig. 1: Block diagram of NTHP-Volterra equalizer.

mode fiber (SMF) with 20G DML and 20G PIN$P D$. The system has an end-to-end 3-dB bandwidth of $15 \mathrm{GHz}$, attaining a sensitivity of $21 \mathrm{dBm}$ at forward error correction (FEC) limit of $1 \times 10^{-2}$. Moreover, $34 \mathrm{~dB}$ power budget can be achieved with an optical booster amplifier (BA) employed at the OLT.

\section{Principle of NTHP-Volterra equalizer}

In order to mitigate inter-symbol interference (ISI) in a bandwidth limited IMDD system, equalizer is used to alter the system's transfer function to be an all-pass filter. FFE-DFE is a typical autoregressive moving-average filter, which can mitigate linear ISI without apparent noise enhancement when there are spectral zeros ${ }^{[7]}$. However, the false hard decision symbols may induce error propagation. To address this issue, the feedback structure of DFE is moved to the transmitter side (Tx), namely linear THP (LTHP) [8]. LTHP-FFE can only compensate linear ISI. Therefore we propose to use NTHP at the Tx and Volterra at the receiver $(\mathrm{Rx})$, referred to as NTHP-Volterra, to further improve the receiver sensitivity by mitigating nonlinear impairments. $A$ block diagram of NTHP-Volterra is shown in Fig. 1. There are mainly three steps for the application of NTHP-Volterra. First, PAM-4 symbols $d(k)$ is transmitted, and Volterra combined with nonlinear decision feedback equalizer (NDFE) (blue box) is used to compensate the linear and nonlinear distortions by using least mean square 


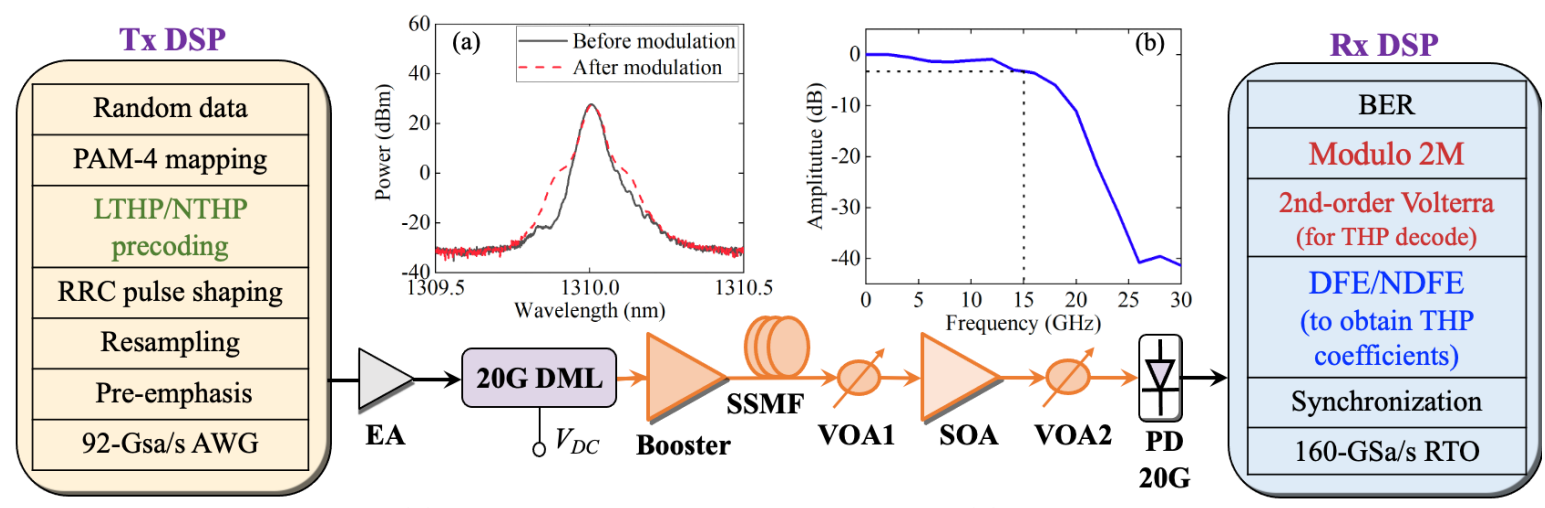

Fig. 2: Experimental setup: (a) spectrum before and after modulation and (b) system end-to-end frequency response.

(LMS) algorithm to minimize $e(k)$. The NDFE module is similar to DFE but replacing linear feedback by a Volterra filter. The coefficients of the Volterra filter are then being used for the NTHP at the Tx. For the second step, the NDFE is moved to the Tx side (green box) by replacing the hard decision module with modulo $2 M$ (for PAM-4, $M$ is 4) which confines its output $x(k)$ between $-2 M$ to $2 M$ with a uniform distribution ${ }^{[9]}$. At the third step, the received signal is equalized by Volterra and another modulo $2 M$ is employed to recover the PAM-4 symbols. For simplicity, only 2nd-order Volterra is used for training during the whole process. To show the performance of NTHP, a comparison with LTHP is conducted in the later section.

\section{Experimental setup}

The system architecture and DSP flow are shown in Fig. 2. At the Tx, the 50 Gbaud PAM-4 signal is generated offline by MATLAB and loaded to an arbitrary waveform generator (AWG, Keysight M8196A, 3-dB bandwidth: $32 \mathrm{GHz}$ ). The output signal is amplified to $1.6 \mathrm{Vpp}$ by an electrical amplifier (EA) with $24 \mathrm{~dB}$ gain before applying on the 20G DML for modulation. The spectrum of optical signal before and after modulation is shown in Fig. 2(a). An O-band booster amplifier (BA) with a maximum output power of $13 \mathrm{dBm}$ is followed to increase the launching power. Then, the $100 \mathrm{~Gb} / \mathrm{s}$ PAM-4 optical signals are transmitted over $20 \mathrm{~km}$ SMF with an average loss of $0.3 \mathrm{~dB} / \mathrm{s}$ at $1310 \mathrm{~nm}$. A variable optical attenuator (VOA1) is applied to emulate the splitter loss and adjust received optical power (ROP) for sensitivity measurement. In order to achieve >29 dB power budget, an O-band semiconductor optical amplifier (SOA) with a noise figure of $7 \mathrm{~dB}$ is used at $\mathrm{Rx}$ before detection. VOA2 is employed to adjust the input power to PIN-PD which is used to detect the signal. The output of PIN-PD is captured by a 160 Gsa/s real-time oscilloscope (RTO, Keysight DSOZ504A, 3-dB bandwidth: $50 \mathrm{GHz}$ ) and processed by offline DSP. The measured system end-to-end frequency response is shown in Fig. 2(b), and the 3-dB bandwidth is around $15 \mathrm{GHz}$, which imposes a major difficulty on supporting 100G PAM-4 transmission. Therefore DSP in both $\mathrm{Tx}$ and $\mathrm{Rx}$ side are used to recovery the signal.

The offline DSP flow at the Tx and Rx side is shown in the yellow and blue flow in Fig. 2, respectively. The random data generated by Mersenne twister are mapped to PAM-4 symbols with a length of $2^{16}$. To compensate ISI induced by the serious bandwidth limitation from the cascaded optoelectrical devices, Nyquist pulse shaping based on root-raised-cosine (RRC) filter is used for $100 \mathrm{~Gb} / \mathrm{s}$ PAM-4 signal. The optimized roll-off factor of RRC is 0.4 . Also, a time-domain digital FIR filter with 5-taps is also employed to pre-emphasis the signal after resampling to match the sampling rate of the AWG. At the Rx side, the captured offline data is resampled by a matched filter, and then synchronization is applied to remove the time jitter and extract the required data. Afterwards, the data is fed into the DFE and Volterra-NDFE equalizer to obtain the linear and nonlinear feedback coefficients for LTHP and NTHP functions at the Tx side. Once NTHP is employed at the Tx side, the 2nd-order Volterra and modulo operation are followed to decode the signal and finally bit error ratio (BER) is calculated.

\section{Experimental results}

The performance of LTHP and NTHP is determined by the coefficients obtained from DFE and NDFE at the Rx side, so we first optimize the taps of FFE-DFE and Volterra-NDFE at the backto-back (BtB) case without precoding at the Tx side. The BER performance versus received optical power is shown in Fig. 3. In Fig. 3(a), with FFE only, the optimized taps length is 47 and it is still hard to achieve LDPD-FEC with a BER of $1 \times 10^{-2}$ at $-16 \mathrm{dBm}$. A further increase of the FFE taps does not improve the performance. After adding DFE, the noise enhancement induced by FFE can be compressed so that the receiver 

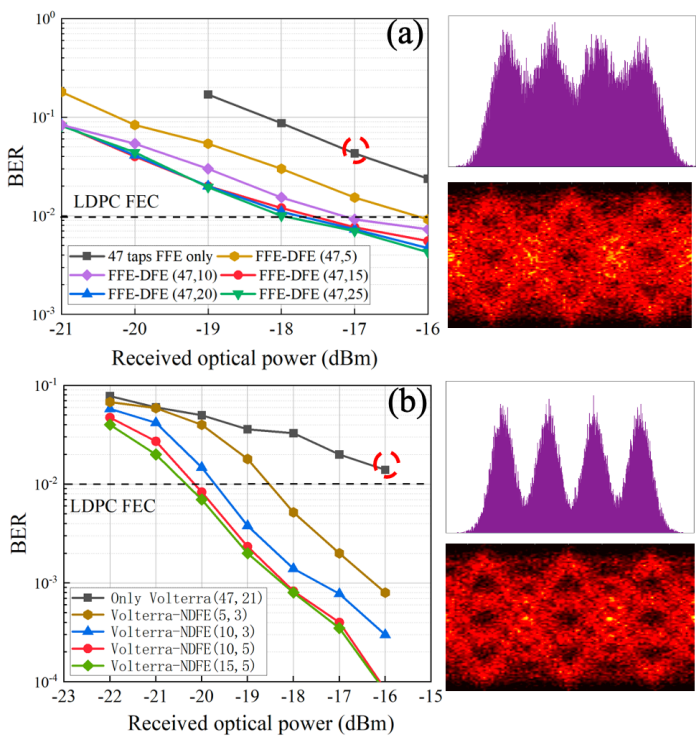

Fig. 3: BER of 100G PAM-4 signal with different taps of (a) DFE and (b) NF-Volterra at BtB, with histograms and eye diagrams measured at the BER circled in red.

sensitivity is improved. As we can see, the optimal DFE tap is 15 , achieving BER of $1 \times 10^{-2}$ at ROP of $-18 \mathrm{dBm}$. The DFE coefficients are kept for LTHP function at the Tx side. For the VolterraNDFE, we also optimize the Volterra taps first, shown in Fig. 3(b). Both Volterra and NDFE are with order-2 kernel considering only first and second order nonlinearity, the lengths of first and second kernel taps are expressed as (L1, L2). For Volterra only, the optimized kernel length is $(47,21)$ which can achieve LDPD-FEC at -16 $\mathrm{dBm}$. We fix this kernel taps and adding NDFE to optimize the kernel taps of NDFE. For NDFE (5, 3 ), about $2.5 \mathrm{dBm}$ sensitivity improvement can be obtained. Further increasing the kernel number continually improves the sensitivity to $-20 \mathrm{dBm}$ until NDFE $(10,5)$. The eye diagram and histogram of PAM-4 signal after FFE and Volterra are shown for reference.

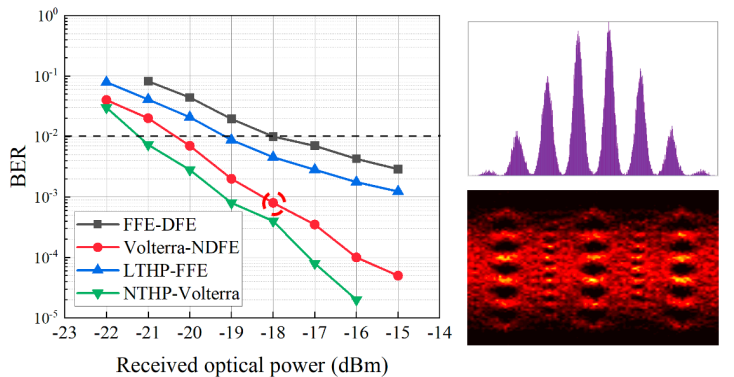

Fig. 4: BER comparison of 100G PAM-4 signal with different equalizers after $20 \mathrm{~km}$ SMF.

With the well-trained feedback coefficients from DFE and NDFE in the Rx, we can then applied it at the Tx side for THP function. The BER comparison of 100G PAM-4 signal after 20 $\mathrm{km}$ SMF transmission with different equalizers is shown in Fig. 4. Since O-band (1310 nm closed to zero dispersion) is employed, the sensitivity

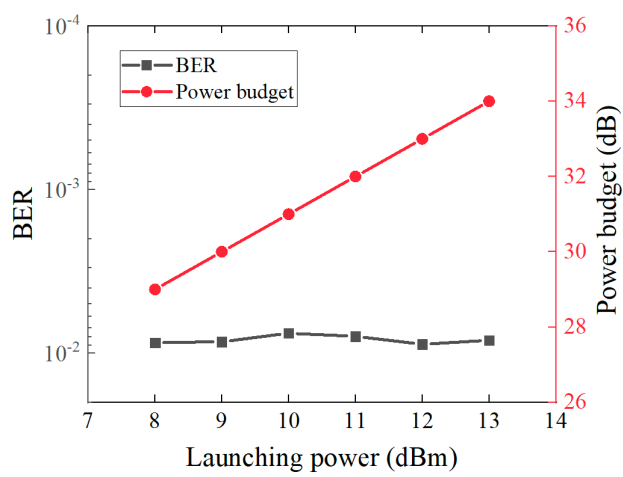

Fig. 5: BER of $100 \mathrm{G}$ PAM-4 signal after $20 \mathrm{~km}$ SMF at -21 $\mathrm{dBm}$ received optical power versus launching power.

after fiber transmission with FFE-DFE and Volterra-NDFE is similar to the BtB case. But compared with FFE-DFE, LTHP-FFE can achieve $1 \mathrm{~dB}$ sensitivity improvement since the error propagation caused by the false decision is avoided at the Tx side. After employing NTHP and 2nd-order Volterra employed, $-21 \mathrm{dBm}$ sensitivity can be obtained. Similar to LTHP, $1 \mathrm{~dB}$ sensitivity improvement compared with VolterraNDFE is obtained. As explained earlier, the modulo $2 M$ with THP in the Tx confines the signal to $-2 M$ to $2 M$ with uniform distribution, therefore in Fig. 4 the histogram and eye diagram with THP looks like multi-level PAM signal. It is recovered to PAM-4 with modulo $2 M$ in the Rx.

Finally, we evaluate the power budget of the system by adjusting the gain of the BA shown in Fig. 5. The receiver sensitivity without BA (-21 $\mathrm{dBm}$ ) is referred to as a benchmark. BER shows small fluctuation after employing the BA when increasing launch power. It is because the DML chirp broaden the spectrum in modulation, which makes the optical signal more tolerant to fiber nonlinearity ${ }^{[10]}$. Thanks to the joint NTHP in the Tx and Volterra in the Rx, a power budget of $34 \mathrm{~dB}$ is obtained with the highest output power of the BA at $13 \mathrm{dBm}$. For PON downstream, NTHP and $B A$ are used in the OLT, whose complexity and cost can be shared by all ONUs.

\section{Conclusions}

We experimentally demonstrate 100G PAM-4 IMDD PON using 20G optics with 3-dB system bandwidth of $15 \mathrm{GHz}$. With the proposed NTHPVolterra algorithm, $2 \mathrm{~dB}$ sensitivity improvement can be obtained compared with LTHP-DFE. As the NTHP is deployed at the OLT side, we can simplify the ONU DSP and achieve a power budget of $34 \mathrm{~dB}$.

\section{Acknowledgements}

This work was supported in part by the National Key R\&D Program of China (2019YFB1803803), Open Fund and the EU H2020 5G STEP-FWD (grant 722429) and POETICS (grant 871769). 


\section{References}

[1] E. Harstead, D. van Veen, V. Houtsma, and P. Dom, "Technology roadmap for time-division multiplexed passive optical networks (TDM PONs)," J. Lightw. Technol., vol. 37, no. 2, pp. 657-664, 2019.

[2] IEEE Standard for Ethernet Amendment 9: Physical Layer Specifications and Management Parameters for $25 \mathrm{~Gb} / \mathrm{s}$ and $50 \mathrm{~Gb} / \mathrm{s}$ Passive Optical Networks, in IEEE Std 802.3ca-2020, 2020, pp. 1-267.

[3] J. Zhang, J. Yu, J. S. Wey, X. Li, L. Zhao, K. Wang, M. Kong, W. Zhou, J. Xiao, X. Xin, and F. Zhao, "SOA Pre-Amplified $100 \mathrm{~Gb} / \mathrm{s} / \lambda$ PAM-4 TDM-PON Downstream Transmission Using 10 Gbps O-Band Transmitters," J. Lightwave Technol., vol. 38, pp. 185193, 2020.

[4] V. Houtsma, E. Chou, and D. van Veen, "92 and 50 Gbps TDM-PON using neural network enabled receiver equalization specialized for PON," in Proc. Opt. Fiber Commun. Conf., 2019, Paper M2B.6.

[5] L. Yi, T. Liao, L. Huang, L. Xue, P. Li, and W. Hu, "Machine learning for $100 \mathrm{~Gb} / \mathrm{s} / \lambda$ passive optical network," J. Lightw. Technol., vol. 37, pp. 1621-1630, 2019.

[6] P. Torres-Ferrera, G. Rizzelli, V. Ferrero, and R. Gaudino, "100+ Gbps/ $\lambda 50$ km C-Band Downstream PON Using CD Digital Pre-Compensation and DirectDetection ONU Receiver," J Lightwave Technol., vol. 38, pp. 6807-6816, 2020.

[7] R. Rath, D. Clausen, S. Ohlendorf, S. Pachnicke, and W. Rosenkranz, "Tomlinson-Harashima Precoding for Dispersion Uncompensated PAM-4 Transmission with Direct-Detection," J Lightwave Technol., vol. 35, pp. 3909-3917, 2017.

[8] S. Luo, Z. Li, Y. Qu, Y. Song, J. Chen, Y. Li and M. Wang, "112-Gb/s/ $\lambda$ Downstream Transmission for TDM-PON with 31-dB Power Budget using 25-Gb/s Optics and Simple DSP in ONU," in Proc. Opt. Fiber Commun. Conf., 2020, Paper Th3k.4.

[9] R. F. H. Fischer, Precoding and Signal Shaping for Digital Transmission, Hoboken, NJ, USA: John Wiley \& Sons, Inc., 2002.

[10] Z. X. Li, L. L. Yi, W. Wei, M. H. Bi, H. He, S. L. Xiao, and W. S. Hu, "Symmetric 40-Gb/s, 100-km Passive Reach TWDM-PON with 53-dB Loss Budget," J Lightwave Technol., vol. 32, pp. 3991-3998, 2014. 